'Sección de Hematología, Hospital del Salvador. Santiago, Chile.

2Laboratorio de Hematología especializada, Hospital del Salvador. Santiago, Chile. ${ }^{3}$ Sección de Hematología. Hospital de Arica. Arica, Chile. aTecnólogo médico.

Recibido el 11 de septiembre de 2018, aceptado el 12 de diciembre de 2018 Correspondencia a: Camila Peña Avenida Salvador 364, Providencia. Santiago, Chile. camipena@gmail.com

\section{Leucemia de células plasmáticas primaria: experiencia en un hospital público chileno}

\author{
MARÍA GABRIELA MENDOZA ${ }^{1}$, XIMENA VALLADARES ${ }^{1}$, \\ MACARENA ROA ${ }^{1}$, MARGARITA REYES $^{2, a}$, ROCÍO OSORIO ${ }^{3}$, \\ MARÍA SOLEDAD UNDURRAGA ${ }^{1}$, MARÍA EUGENIA LEGÜES ${ }^{2, a}$, \\ MARÍA ELENA CABRERA ${ }^{1}$, CAMILA PEÑA ${ }^{1}$
}

\section{Primary plasma cell leukemia. Report of five cases}

Background: Primary plasma cell leukemia ( $p P C L$ ) is uncommon, aggressive and has a different biology than multiple myeloma (MM). Aim: To report the features of patients with PPCL. Material and Methods: Review of databases of the Hematology Department and the Hematology laboratory. Results: Of 178 patients with monoclonal gammopathies, five (2.8\%) patients aged 33 to 64 years (three females) had a pPCL. The mean hemoglobin was $7.3 \mathrm{~g} / \mathrm{dL}$, the mean white blood cell count was 52,500/ $\mathrm{mm}^{3}$, with $58 \%$ plasma cells, and the mean platelet count was $83,600 / \mathrm{mm}^{3}$. The mean bone marrow infiltration was 89\%, LDH was 2,003 IU/L, serum calcium was $13 \mathrm{mg} / \mathrm{dL}$, and creatinine 1.5 $\mathrm{mg} / \mathrm{dL}$. Two patients had bone lesions. Three were IgG, one IgA lambda and one lambda light chain. CD20 was positive in one, CD56 was negative in all and CD117 was negative in 3 cases. By conventional cytogenetic analysis, two had a complex karyotype. By Fluorescence in situ Hybridization, one was positive for TP53 and another for $t(11 ; 14)$. One patient did not receive any treatment, three patients received VTD PACE and one CTD. None underwent transplant. Three patients are alive. The mean survival was 14 months. Conclusions: These patients with pPCL were younger and had a more aggressive clinical outcome than in multiple myeloma.

(Rev Med Chile 2019; 147: 18-23)

Key words: Bortezamib; Leukemia, Plasma Cell; Paraproteinemias.
L a leucemia de células plasmáticas (LCP) es una neoplasia de células plasmáticas infrecuente y agresiva, caracterizada por la presencia de altos recuentos de células plasmáticas circulantes en sangre periférica. Fue descrita inicialmente por Gluzinski hace más de 100 años $^{1} \mathrm{y}$ definida por Kyle en $1974^{2}$. La IMWG (del inglés International Myeloma Working Group) define a la LCP como la presencia de plasmocitos en sangre periférica en $>20 \%$ del total de células nucleadas o al recuento absoluto de células plasmáticas en sangre periférica $>2 \times 10^{9} / \mathrm{L}^{3}$.
Se presenta en 1-2\% de todas las gammapatías monoclonales $^{4}$ en dos formas: LCP primaria y secundaria. La LCP primaria (LCPp) representa 60-70\% de los casos. Esta se presenta "de novo"en fase leucémica, sin evidencia previa de mieloma múltiple (MM), y difiere de la LCP secundaria, precisamente, en que esta se refiere a "transformación leucémica" en pacientes con MM previamente diagnosticados, refractarios o resistentes. $\mathrm{Su}$ evolución es fulminante, con sobrevida sin tratamiento muy corta, entre 9 y 11 semanas ${ }^{5}$.

En vista de la baja frecuencia de esta enfer- 
medad, la mayoría de los datos clínicos se han obtenido de casos aislados reportados y estudios retrospectivos pequeños.

Se encontró solo un reporte a nivel nacional de LCPp, correspondiente a un caso clínico ${ }^{6}$.

El objetivo de esta investigación fue describir las características epidemiológicas, clínicas, respuesta a tratamiento y sobrevida de pacientes mayores de 15 años con diagnóstico de LCPp, diagnosticados y tratados en el Hospital del Salvador.

\section{Materiales y Métodos}

\section{Pacientes}

Se realizó un estudio de tipo descriptivo, retrospectivo. Los datos fueron extraídos desde la base de datos de la Sección de Hematología y de la base del Laboratorio de Hematología Especializada del Hospital del Salvador. Se buscaron los pacientes con diagnóstico de LCPp, entre enero de 2015 y abril de 2018. Se excluyó del análisis los pacientes con LCP secundaria y pacientes con diagnóstico de cualquier otra gammapatía monoclonal que no cumplieran los criterios de LCPp.

\section{Epidemiología, clínica y laboratorio}

Se revisó la base de datos clínica y de laboratorio, además de las fichas clínicas de los pacientes con LCPp. Se registraron los antecedentes clínicos como edad al diagnóstico, género, comorbilidades y sintomatología; además, antecedentes de laboratorio como hemograma, porcentaje de células plasmáticas en sangre periférica y médula ósea, estudio de paraproteína, niveles de LDH, calcio, creatinina, B2 microglobulina y albúmina. La afectación ósea se obtuvo mediante radiografías convencionales en 2 pacientes y con tomografía computada sin contraste en 3 . También se registró el estudio de citometría de flujo y citogenética e hibridación in situ con fluorescencia (FISH).

\section{Citogenética convencional y FISH}

Se realizaron cultivos de 24 a 96 h, analizándose 20 metafases como mínimo. Las alteraciones cromosómicas fueron descritas con la nomeclatura aceptada internacionalmente. Se realizó FISH interfásico a las células en fijador provenientes del cultivo de citogenética, con sondas comerciales Vysis (Abbott Downers Grove, IL, USA) para $\operatorname{del}(17 \mathrm{p}), \mathrm{t}(4 ; 14), \mathrm{t}(11 ; 14)$ y $\mathrm{t}(14 ; 16)$.

\section{Inmunofenotipo por citometría de flujo}

Todas las muestras fueron marcadas según protocolo de Euroflow "LST" de 8 colores. El estudio se realizó en equipo BD FACSCanto II (BD Bioscience, Becton, Dickinson and Company, USA) con 3 láseres (azul, violeta y rojo), y fue adquirido y analizado con software BD FACSDiva (Becton, Dickinson and Company, USA).

\section{Estadística}

Se realizó estadística descriptiva con los datos obtenidos.

El estudio fue aprobado por el Comité de Ética del Servicio de Salud Metropolitano Oriente.

\section{Resultados}

De un total de 178 pacientes con diagnóstico, de gammapatía monoclonal en el período de estudio, se diagnosticó 8 pacientes con LCP, cinco de ellas primaria $(2,8 \%)$. La edad promedio al diagnóstico fue $52 \pm 13$ años, (rango 33-64). La relación hombre:mujer fue de 1:1,5.

Tres presentaron hipercalcemia al diagnóstico con un valor medio de calcio sérico de 13,1 $\pm 4,0$ $\mathrm{mg} / \mathrm{dL}$; falla renal (definido como creatinina $>2$ $\mathrm{g} / \mathrm{dL}$ ) en $60 \%$, con creatinina media $1,48 \pm 0,72$ $\mathrm{g} / \mathrm{dL}$. Todos presentaron anemia. La hemoglobina $(\mathrm{Hb})$ media fue $7,3 \pm 2,8 \mathrm{mg} / \mathrm{dL}$, el recuento de leucocitos medio fue $52.500 \pm 38.056 / \mathrm{mm}^{3} \mathrm{y}$ el recuento plaquetario medio $83.600 \pm 55.936 /$ $\mathrm{mm}^{3}$ (Tabla 1). Se identificó compromiso óseo solo en $2 / 5$ casos. En otros estudios bioquímicos, $80 \%$ de los pacientes presentó elevación de B2 microglobulina, media $12,3 \pm 7,7 \mathrm{mg} / \mathrm{dl} ; 40 \%$ de los casos presentó hipoalbuminemia y la mediana de deshidrogenasa láctica (LDH) fue $2.063 \mathrm{UI} / \mathrm{mL}$ (normal hasta $224 \mathrm{UI} / \mathrm{mL}$ ).

El recuento promedio de células plasmáticas en sangre periférica fue $57,6 \%$, y en médula ósea $88,6 \%$. Con respecto a la paraproteína, se observaron 3 casos IgG (2 IgG kappa, 1 IgG lambda), 1 caso IgA lambda y un caso de cadenas livianas lambda.

El inmunofenotipo se realizó en cuatro pacientes. Destacó que CD56 fue negativo en todos, CD117 negativo en tres y el CD20 positivo en uno (Tabla 2).

Se realizó estudio por citogenética convencional en cuatro pacientes, dos presentaron un 
Tabla 1. Características generales del grupo

\begin{tabular}{|c|c|c|}
\hline Características & ítem & n (\%) \\
\hline \multirow[t]{10}{*}{ Epidemiología } & Edad (años) & $\begin{array}{c}52 \\
\text { (rango 33-64) }\end{array}$ \\
\hline & Masculino & $2(40)$ \\
\hline & Laboratorio Hb (g/dL) & $7,3 \pm 2,8$ \\
\hline & $\mathrm{GB}\left(\mathrm{mm}^{3} \times 10^{9}\right)$ & $52,5 \pm 38,0$ \\
\hline & Plaquetas $\left(\mathrm{mm}^{3} \times 10^{9}\right)$ & $83,6 \pm 55,9$ \\
\hline & $\mathrm{LDH}(\mathrm{UI} / \mathrm{mL})$ & 2060 \\
\hline & Calcio (mg/dL) & $13,1 \pm 4,0$ \\
\hline & Creatinina (mg/dL) & $1,48 \pm 0,72$ \\
\hline & B2 microglobulina (mg/L) & $12,3 \pm 7,7$ \\
\hline & Albúmina (g/L) & $3,65 \pm 1,06$ \\
\hline \multirow{2}{*}{$\begin{array}{l}\text { Células } \\
\text { plasmáticas }\end{array}$} & Sangre periférica & $57,629,1$ \\
\hline & Médula ósea & $88,6 \pm 4,7$ \\
\hline \multirow[t]{4}{*}{ Paraproteína } & lgG kappa & $2(40)$ \\
\hline & lgG lambda & $1(20)$ \\
\hline & $\lg A$ lambda & $1(20)$ \\
\hline & CL lambda & $1(20)$ \\
\hline
\end{tabular}

Tabla 2. Estudio de citometría de flujo en cuatro casos estudiados

\begin{tabular}{|ccccc|}
\hline Anticuerpo & caso 1 & caso $\mathbf{3}$ & caso 4 & caso $\mathbf{5}$ \\
$\mathrm{CD} 38$ & + & + & + & + \\
$\mathrm{CD} 138$ & + & + & + & + \\
$\mathrm{CD} 20$ & - & - & + parcial & - \\
$\mathrm{CD} 19$ & - & - & - & - \\
$\mathrm{CD} 117$ & + & - & & - \\
$\mathrm{CD} 56$ & - & - & - & - \\
\hline
\end{tabular}

Nota: caso 2 no fue estudiado.

cariotipo complejo, uno fue normal y uno sin mitosis. Los cariotipos complejos tuvieron un promedio de 15 20 alteraciones cromosómicas/ célula (Figura 1). Los cinco casos tuvieron estudio de FISH, uno con resultado positivo para TP53 y uno para $\mathrm{t}(11 ; 14)$. No se detectó $\mathrm{t}(4 ; 14)$ ni $\mathrm{t}(14 ; 16)$ (Tabla 3).

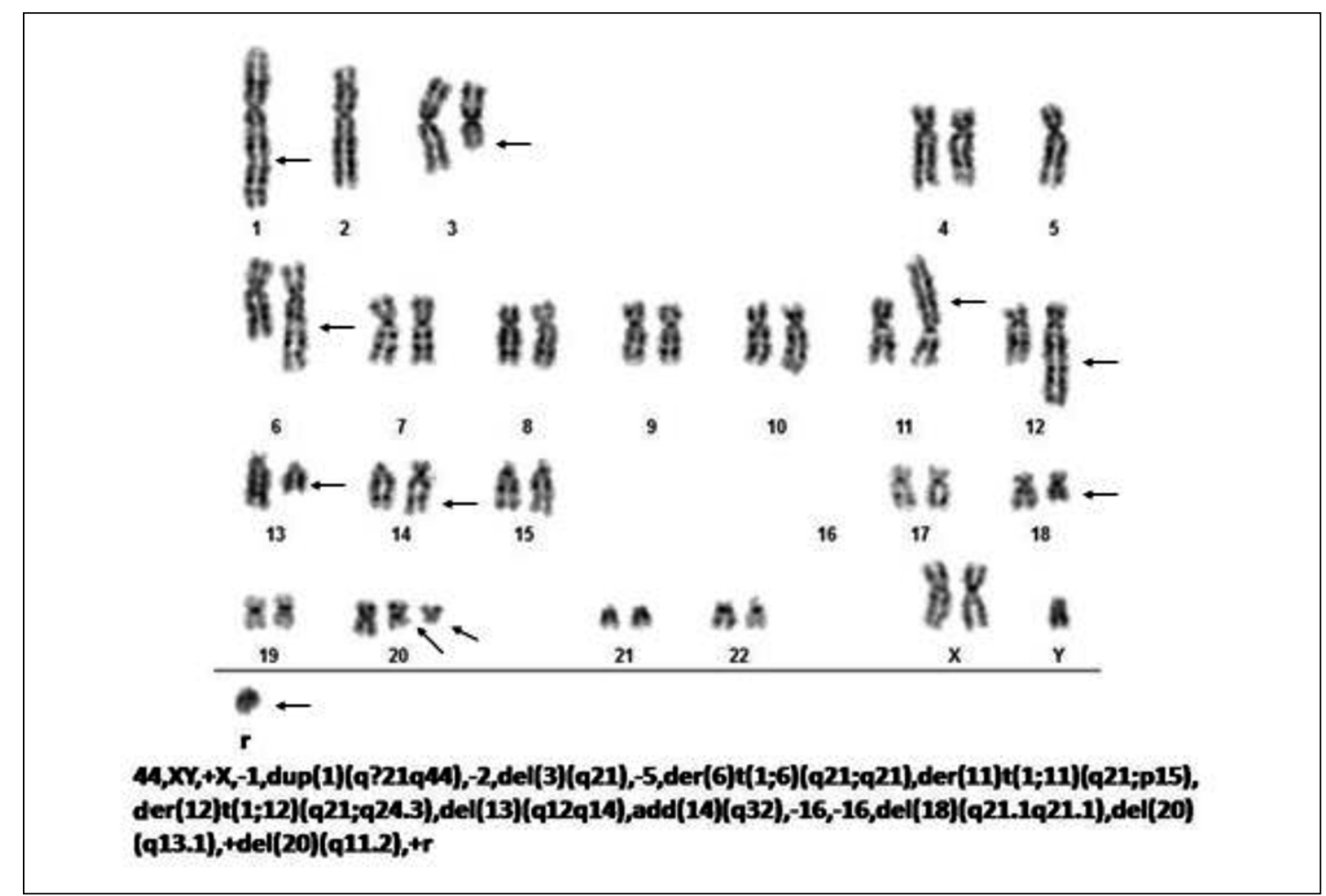

Figura 1. Cariotipo del caso 1 mostrando una hipodiploidía con alteraciones cromosómicas complejas. Destacan las copias extra de 1q participando en translocaciones, deleción de 13q, material adicional en 14q y ausencia de los cromosomas 16. 
Tabla 3. Resultados del estudio citogenético y FISH de los 5 casos

\begin{tabular}{|c|c|c|c|c|c|}
\hline Caso & Cariotipo & TP53 & $t(4 ; 14)$ & $t(14 ; 16)$ & $t(11 ; 14)$ \\
\hline 1 & Complejo & $\mathrm{N}$ & $\mathrm{N}$ & $\mathrm{N}$ & $\mathrm{N}$ \\
\hline 2 & Norma I & Positivo & $\mathrm{N}$ & $\mathrm{N}$ & $\mathrm{N}$ \\
\hline 3 & Complejo & $\mathrm{N}$ & $\mathrm{N}$ & $\mathrm{N}$ & $\mathrm{N}$ \\
\hline 4 & NA & $\mathrm{N}$ & $\mathrm{N}$ & $\mathrm{N}$ & $\mathrm{N}$ \\
\hline 5 & No mitosis & $\mathrm{N}$ & $\mathrm{N}$ & $\mathrm{N}$ & Positivo \\
\hline
\end{tabular}

En cuanto a tratamiento, tres recibieron esquema VTD PACE (bortezomib, dexametasona en altas dosis, talidomida, cisplatino, doxorrubicina, ciclofosfamida y etopósido) por 4 ciclos, un paciente abandonó la atención tras un ciclo de CTD (ciclofosfamida, talidomida y dexametasona), y el otro no recibió tratamiento, ya que falleció al segundo día de hospitalización. Ninguno ha recibido trasplante de progenitores hematopoyéticos (TPH). De los 3 pacientes que tenemos datos, 2 lograron remisión completa, recayendo a los 5 y 6 meses, respectivamente. Uno tuvo respuesta parcial. Tres permanecen vivos, con enfermedad activa, en manejo conservador. La sobrevida media fue de 14 meses.

\section{Discusión}

La LCPp es una entidad única, poco frecuente, con características biológicas particulares ${ }^{7}$. En nuestro estudio observamos $4,4 \%(8 / 178)$ leucemia de células plasmáticas y 2,8\% (5/178) de LCPp, acorde a lo reportado a nivel internacional. La edad media al diagnóstico de las LCPp fue 52 años, confirmando que son pacientes de menor edad que los con MM. A diferencia de lo reportado en la literatura, evidenciamos mayor incidencia en sexo femenino, hecho que concuerda con un estudio nacional realizado por Peña et $\mathrm{al}^{8}$, que muestra una frecuencia mayor de MM en mujeres. Por otro lado, observamos que solo un paciente se asoció a LCPp por cadenas livianas, a diferencia de lo descrito. Sin perjuicio de lo anterior, predominó la presencia de cadena lambda, hecho que coincide con lo reportado internacionalmente .

Se postula que el hecho que las células plasmáticas dejen la médula ósea para migrar a la sangre periférica se explica por la ausencia de expresión en membrana de moléculas de adhesión $(\mathrm{CD} 56)^{10,11}$. Genzel et $\mathrm{al}^{9}$ reportaron recientemente presencia de CD56 solo en $29 \%$ de los casos de LCPp vs 70\% en el caso del MM. En nuestro estudio, ningún paciente expresó CD56.

Tal como esperábamos, observamos que los pacientes con LCPp se presentaron con alta incidencia de falla renal e hipercalcemia y menos frecuentemente lesiones óseas ${ }^{12}$. No se encontró lesiones de sistema nervioso central, aunque cabe destacar que esto no fue activamente buscado.

El estudio inmunofenotípico por citometría de flujo de las LCPp difiere de la del MM por presentar expresión de CD20 intenso, expresión débil de CD19, CD117, HLA-DR y ausencia de CD56 hasta en $80 \%$ de los $\operatorname{casos}^{11,13}$. Nosotros observamos expresión parcial de CD20 en un paciente, CD56 negativo en todos y CD117 negativo en 3 casos. Como ya se mencionó, no se expresó CD56 en ninguno.

En relación a los hallazgos del estudio citogenético, se evidenció alta frecuencia de mitosis y cariotipos complejos. Esto se condice con la mayor tasa de replicación celular de esta entidad. Todas las alteraciones citogenéticas encontradas en MM pueden ser encontradas en las LCP, teniendo alta prevalencia la $\mathrm{t}(11 ; 14)$, monosomía 13 o del(13) y monosomía $7^{14,15}$. Destaca la del(17) con pérdida de TP53 hasta 50\% de los casos de LCPp, hecho que observamos en un solo paciente. La t $(11 ; 14)$ es frecuente y está asociada a mal pronóstico en esta patología, a diferencia del MM, donde se asocia a pronóstico favorable ${ }^{15,16}$.

Aunque con la introducción de las nuevas terapias para MM (talidomida, lenalidomida o bortezomib), los resultados en cuanto a sobrevida de estos pacientes con LCPp mejoraron, siguen siendo poco alentadores. Un estudio estadounidense sobre la base de datos SEER (del inglés Sur- 
veillance, Epidemiology, and End Results database) publicó en 2009 resultados de esta patología desde los años 1973 y 2004. La sobrevida media fue de 4 meses en pacientes mayormente tratados con quimioterapia convencional. Lamentablemente, no hubo ninguna mejoría de sobrevida durante los 30 años ${ }^{17}$. En la actualización publicada en 2014, se observó un incremento en sobrevida significativa en los pacientes tratados luego del 2006, la que llegó a 12 meses entre 2006 y $2009^{18}$. La explicación más probable es el uso masificado de las llamadas nuevas drogas.

Por otro lado, se ha reportado disminución en la mortalidad en el primer mes del diagnóstico, que alcanzaba $26 \%$ antes de 2006 , a $15 \%$ en Estados Unidos de Norteamérica. Esto podría extrapolarse a nuestra cohorte, en la que hubo 1 fallecido en el primer mes.

En general, el bortezomib es la droga mayormente usada para esta patología ${ }^{19-22}$, aunque no hay esquema estándar.

Desde la introducción de la quimioterapia en altas dosis, seguido por trasplante de médula autólogo $(\mathrm{TAPH})$ en pacientes candidatos ${ }^{23}$, se observó una mejoría significativa en la sobrevida de pacientes con mieloma múltiple. Sin embargo, los resultados no son extrapolables a los casos de LCPp, en los que la sobrevida es francamente menor $^{24-28}$. No hay estudios prospectivos fase III, por lo que no existen guías precisas de tratamiento. Solo existen 2 estudios fase II $^{19,29}$. Uno evaluó 23 pacientes menores de 70 años, con tratamiento basado en bortezomib, dexametasona más ciclofosfamida o doxorrubicina, seguido de TAPH. Algunos recibieron trasplante alogénico de consolidación, otros un segundo TAPH. La sobrevida media fue de 36 meses, con una tasa de respuesta a la inducción de $69 \%$. El otro estudio evaluó la combinación de lenalidomida y dexametasona como inducción en 23 pacientes. La sobrevida a 15 meses de seguimiento fue de $63 \%$, con tasa de respuesta de $61 \%$. Sin perjuicio de lo anterior, las recomendaciones actuales recomiendan un régimen intensivo, que incluya nuevas terapias y quimioterapia, seguido de $\mathrm{TAPH}^{7,30}$.

En nuestros pacientes, se eligió el esquema VTD PACE por ser agresivo y usado en MM de alto riesgo o enfermedad extramedular ${ }^{31}$. Fue efectivo en los tres pacientes en que se usó, sin embargo, todos recayeron rápidamente. Lamentablemente, ninguno logró ser candidato a TAPH, ya que esta enfermedad no se incluye dentro de las indicaciones actuales de la Comisión de Trasplante del sistema público chileno.

\section{Conclusión}

La leucemia de células plasmáticas es una patología infrecuente y agresiva. Nuestros pacientes presentaron similares características a las descritas en la literatura. Aunque el pronóstico ha mejorado, este sigue siendo ominoso, con recaídas tempranas a pesar de la introducción de quimioterapia intensiva. El ideal es proceder rápidamente a un TAPH, una vez lograda la remisión de la enfermedad.

\section{Referencias}

1. Gluzinski A, Reichentein M. Myeloma und leucaemia lymphatica plasmocellularis. Wien Klin Wochenschr 1906; 19: 336.

2. Kyle RA, Maldonado JE, Bayrd ED. Plasma cell leukemia. Report on 17 cases. Arch Intern Med 1974; 133 (5): 813-8.

3. Fernández de Larrea C, Kyle RA, Durie BGM, Ludwig H, Usmani S, Vesole DH, et al. Plasma cell leukemia: consensus statement on diagnostic requirements, response criteria and treatment recommendation by the International Myeloma Working Group. Leukemia 2013; 27: 780-91.

4. McKenna RW, Kyle RA, Kuehl WM, Harris NL, Coupland RW, Fend F. Plasma Cell neoplasms. In: Swerdlow SH, Campo E, Lee Harris N, Jaffe ES, Pileri SA, Stein $\mathrm{H}$, Thiele J. WHO Classification of tumors of Haematopoietic and Lymphoid Tissues: 239-56. Revised 4th Ed. International Agency for Research on Cancer, 2016. Lyon. France. p 585.

5. Musto P, Simeon V, Todoerti K, Neri A. Primary Plasma Cell Leukemia: Identity Card 2016. Curr Treat Options Oncol 2016; 17 (4): 19.

6. Yáñez J, Saldías V, Díaz O, Saldías F. Compromiso pleural en la leucemia de células plasmáticas. Reporte de un caso. Rev Chil Enf Respir 2008; 24: 304-8.

7. Gavriatopoulou M, Musto P, Caers J, Merlini G, Kastritis E, van de Donk N, et al. European myeloma network recommendations on diagnosis and management of patients with rare plasma cell dyscrasias. Leukemia 2018; 32 (9): 1883-98.

8. Peña C, Rojas C, Rojas H, Soto P, Cardemil D, Aranda $\mathrm{S}$, et al. Mieloma múltiple en Chile: pasado, presente y 
futuro del programa nacional de drogas antineoplásicas (PANDA). Revisión de 1.103 pacientes. Rev Med Chile 2018; 146: 869-75.

9. Ganzel C, Rouvio O, Avivi I, Magen H, Jarchowsky O, Herzog K, et al. Primary plasma cell leukemia in the era of novel agents for myeloma-multi center retrospective analysis of outcome. Leukemia Research 2018; 68: 9-14.

10. Pellat-Deceunynck C, Barillé S, Jego G, Puthier D, Robillard N, Pineau D, et al. The absence of CD56 (NCAM) on malignant plasma cells is a hallmark of plasma cell leukemia and of a special subset of multiple myeloma. Leukemia 1998; 12 (12): 1977-82.

11. García-Sanz R, Orfão A, González M, Tabernero MD, Bladé J, Moro MJ, et al. Primary plasma cell leukemia: clinical, immunophenotypic, DNA ploidy, and cytogenetic characteristics. Blood 1999; 93 (3): 1032-7.

12. Dimopoulos MA, Palumbo A, Delasalle KB, Alexanian R. Primary plasma cell leukaemia. Br J Haematol 1994; 88 (4): 754-9.

13. Kraj M, Kopeć-Szlęzak J, Pogłód R, Kruk B. Flow cytometric immunophenotypic characteristics of 36 cases of plasma cell leukemia. Leuk Res 2011; 35 (2): 169-76.

14. Neri A, Todoerti K, Lionetti M, Simeon V, Barbieri M, Nozza F, et al. Primary plasma cell leukemia 2.0: advances in biology and clinical management. Expert Rev Hematol 2016; 9: 1063-73.

15. Avet-Loiseau H, Roussel M, Campion L, Leleu X, Marit G, Jardel H, et al. Cytogenetic and therapeutic characterization of primary plasma cell leukemia: the IFM experience. Leukemia 2012; 26: 158-9.

16. Usmani SZ, Nair B, Qu P, Hansen E, Zhang Q, Petty N, et al. Primary plasma cell leukemia: clinical and laboratory presentation, gene-expression profiling and clinical outcome with Total Therapy protocols. Leukemia 2012; 26 (11): 2398-405.

17. Ramsingh G, Mehan P, Luo J, Vij R, Morgensztern D. Primary plasma cell leukemia: a surveillance, epidemiology, and end results database analysis between 1973 and 2004. Cancer 2009; 115 (24): 5734-9.

18. Gonsalves WI, Rajkumar SV, Go RS, Dispenzieri A, Gupta V, Singh PP, et al. Trends in survival of patients with primary plasma cell leukemia: a population-based analysis. Blood 2014; 124 (6): 907-12.

19. Royer B, Minvielle S, Diouf M, Roussel, Karlin L, Hulin C, et al. Bortezomib, doxorubicin, cyclophosphamide, dexamethasone induction followed by stem cell transplantation for primary plasma cell leukemia: a prospective phase II study of the Intergroupe Francophone du Myelome. J Clin Oncol 2016; 34: 2125-32.
20. D’Arena G, Valentini CG, Pietrantuono G, Guariglia R, Martorelli MC, Mansueto G, et al. Frontline chemotherapy with bortezomib-containing combinations improves response rate and survival in primary plasma cell leukemia: a retrospective study from GIMEMA Multiple Myeloma Working Party. Ann Oncol 2012; 23: 1499-502.

21. Katodritou E, Terpos E, Kelaidi C, Kotsopoulou M, Delimpasi S, Kyrtsonis MC, et al. Treatment with bortezomib-based regimens improves overall response and predicts for survival in patients with primary or secondary plasma cell leukemia: analysis of the Greek myeloma study group. Am J Hematol 2014; 89: 145-50.

22. Lebovic D, Zhang L, Alsina M, Nishiroti T, Shain KH, Sullivan D, et al. Clinical outcomes of patients with plasma cell leukemia in the era of novel therapies and hematopoietic stem cell transplantation strategies: a single-institution experience. Clin Lymphoma Myeloma Leuk 2011; 11: 507-11.

23. Child JA, Morgan GJ, Davies FE, Owen RG, Bell SE, Hawkins K, et al. High-dose chemotherapy with hematopoietic stem- cell rescue for multiple myeloma. $\mathrm{N} \mathrm{Engl}$ J Med 2003; 348 (19): 1875-83.

24. Musto P. Progress in the treatment of primary plasma cell leukemia. J Clin Oncol 2016; 34: 2082-4.

25. Mahindra A, Kalaycio ME, Vela-Ojeda J, Vesole DH, Zhang MJ, Li P, et al. Hematopoietic cell transplantation for primary plasma cell leukemia: results from the Center for International Blood and Marrow Transplant Research. Leukemia 2012; 26: 1091-7.

26. Nishihori T, Abu Kar SM, Baz R, Alsina M, Harousseau JL, Kharfan-Dabaja MA. Therapeutic advances in the treatment of primary plasma cell leukemia: a focus on hematopoietic cell transplantation. Biol Blood Marrow Transplant 2013; 19: 1144-51.

27. Jurczyszyn A, Radocha J, Dávila J, Fiala MA, Gozzetti A, Grząśko N, et al. Prognostic indicators in primary plasma cell leukaemia: a multicentre retrospective study of 117 patients. Br J Haematol 2018; 180 (6): 831-9.

28. Musto P, Simeon V, Martorelli MC, Petrucci MT, Cascavilla N, Di Raimondo F, et al. Lenalidomide and low-dose dexamethasone for newly diagnosed primary plasma cell leukemia. Leukemia 2014; 28: 222-5.

29. van de Donk NW, Lokhorst HM, Anderson KC, Richardson PG. How I treat plasma cell leukemia. Blood 2012; 120: 2376-89.

30. Kapoor P, Ramakrishnan V, Rajkumar SV. Bortezomib combination therapy in multiple myeloma. Semin Hematol 2012; 49 (3): 228-42. 